\title{
TREINAMENTO COM PESOS E INDICADORES DE AGILIDADE DE MULHERES IDOSAS
}

\author{
Miguel Soares Conceição \\ Valéria Bonganha \\ Fábio Bitencourt Leivas \\ Giancarlo B. M. Bruno \\ Felipe Cassaro Vechin \\ Mara Patrícia Traina Chacon-Mikahil \\ Vera Aparecida Madruga
}

O presente estudo teve como objetivo analisar os efeitos de diferentes tipos de treinamentos sobre o nível de agilidade de mulheres idosas. Foram selecionadas 42 mulheres idosas saudáveis e ativas fisicamente, divididas em três grupos: grupo treinamento de força $(\mathrm{GTF}, \mathrm{n}=14)$, grupo resistência muscular localizada (GRML, $\mathrm{n}=14)$, e grupo controle $(\mathrm{GC}, \mathrm{n}=14)$. Os programas tiveram duração de 12 semanas, com freqüência de três sessões em dias alternados. $\mathrm{O}$ indicador de agilidade foi avaliado por meio de um teste de agilidade adaptado da AAHPERD. Os resultados mostram diferença estatisticamente significante no indicador de agilidade somente para o GTF, o GRML melhorou o tempo obtido no teste, embora de maneira não significativa. Podemos concluir que o treinamento de força parece ter influenciado preterivelmente a agilidade de mulheres idosas, podendo contribuir para a manutenção da autonomia e da agilidade desta população.

\section{Palavras-Chave}

Treinamento com pesos; Atividades da vida diária, Idosos.

\section{EFFECTS OF STRENGTH TRAINING ON AGILITY IN OLDER WOMEN}

This study had as aim to analyze the effects of different types of physical training in the agility levels of elderly women. It was selected 42 healthy and active elderly women divided into three groups: group of strength training $(\mathrm{GTF}, \mathrm{n}=14)$, group of local muscular resistance (GRML, $\mathrm{n}=14$ ), and control group (GC, $\mathrm{n}=14$ ). The programs last 12 weeks, with a frequency of three times a week, in alternate days. The agility marker was evaluated using an adapted agility test from AAHPERD. The results showed significant statistical difference to the agility marker only to the GTF, the GRML improved the time obtained in the test, although in a no significant way. We can conclude that the strength training seems to better improve the agility markers of elderly women, and can contribute to maintain the autonomy and the agility of this population.

\section{Key-Words}

Strength training; Life diary activities; Elderly. 


\section{INTRODUÇÃO}

A longevidade da população mundial vem crescendo significantemente nas últimas décadas de acordo com pesquisas realizadas pelos principais órgãos ligados à saúde (ACSM, 2003; WHO, 2005), revelando uma maior preocupação com a diminuição da autonomia funcional no envelhecimento.

No ano de 2002 no Brasil, o número de pessoas com mais de 60 anos era cerca de 16 milhões, tendo uma representatividade de 9,3\% da população brasileira. Em 2020 é possível que este número se eleve para 25 milhões de idosos, ou seja, 11,4\% da população (IBGE, 2004) e dessa maneira, ganha ênfase a preocupação com a qualidade e o avançar dos anos de vida da população.

Desta forma, observa-se que a perda da massa muscular e, conseqüentemente, da força muscular é um dos principais responsáveis pela deterioração na mobilidade e na capacidade funcional do indivíduo mais velho (MATSUDO et al., 2003). A incapacidade funcional define-se pela presença de dificuldade no desempenho de certos gestos e de certas atividades da vida cotidiana ou mesmo pela impossibilidade de desempenhá-las (ROSA et al., 2003).

O indivíduo idoso, praticante de atividade física regular pode manter ou melhorar os níveis da capacidade cardiorrespiratória, da flexibilidade, da coordenação, da força e da agilidade, sendo esta última, uma capacidade que desempenha um papel fundamental para a locomoção. $\mathrm{O}$ desenvolvimento ou a manutenção de bons níveis de agilidade contribuem para a diminuição do risco de acidentes, evitando que fatores adicionais venham a atuar de forma negativa junto ao processo de envelhecimento (FERREIRA; GOBBI, 2003).

A agilidade, em conjunto com as demais capacidades físicas, pode proporcionar maior independência do indivíduo na terceira idade minimizando assim sua limitação e dependência física.

Quando há um comprometimento da capacidade funcional, o indivíduo passa a necessitar de ajuda, principalmente da família para realizar as atividades da vida diária. Além deste fato há também uma grande sobrecarga ao sistema de saúde (GURALNIK; LACROIX, 1992) ${ }^{7}$.

Diante deste quadro, o interesse de pesquisadores na investigação das causas e mecanismos envolvidos na diminuição da capacidade funcional com o avançar da idade tem aumentado, buscando a criação de estratégias para minimizar as perdas fisiológicas decorrentes do processo de envelhecimento.

Conexões: revista da Faculdade de Educação Física da UNICAMP, Campinas, v. 8, n. 2, p. 68-82, maio/ago. 2010.

ISSN: 1983-9030. 
As medidas de prevenção ou redução da magnitude dos efeitos do envelhecimento estão diretamente ligadas, e associadas as medidas gerais de saúde e programas de atividade física. Este fato tem sido discutido não somente nos chamados países desenvolvidos ou do primeiro mundo, como também nos países em desenvolvimento, como é o caso do Brasil (MATSUDO, 2000).

Uma das preocupações relacionadas as perdas no envelhecimento é com a sarcopenia, ou seja, a diminuição da massa muscular, que está associada às alterações ósteomusculares que são significativas para a qualidade de vida e independência dos indivíduos em fase de envelhecimento, pois acarretam déficit no andar e na mobilidade, e conseqüentemente, dificuldades em realizar tarefas comuns do cotidiano. Estima-se que $25 \%$ dos idosos com menos de 70 anos são acometidos por sarcopenia (BAUMGARTNER et al., 1998).

Associado a perda da massa muscular observa-se importante redução nos níveis de força, que estão associados à menor velocidade de caminhada e à inaptidão física que acarretaria na elevação do risco de quedas e fraturas na população idosa (LATHAM et al., 2004).

Baseado no fato da agilidade estar relacionada diretamente com outras capacidades como força muscular, flexibilidade e velocidade, que sofrem uma redução em seus níveis com a idade, a probabilidade desta acompanhar este declínio é muito grande.

Para avaliação das capacidades físicas a American Alliance for Health, Physical Education, Recreation and Dance (AAHPERD) propôs uma bateria de testes específica para medir a aptidão funcional em idosos, composta de cinco testes motores (coordenação, resistência de força, flexibilidade, agilidade, resistência aeróbia geral).

A avaliação da agilidade pode ser realizada nos segmentos corporais isoladamente, ou de uma forma geral, sendo que a agilidade geral permite uma melhor locomoção, mas ambas fundamentais para manutenção da qualidade de vida dos indivíduos na terceira idade (FERREIRA, GOBBI, 2003).

Programas de treinamento como, os resistidos ou com pesos, tem se mostrado efetivo no controle da manutenção da força muscular e no equilíbrio da massa óssea, atuando na capacidade de locomoção e na prevenção de fraturas, principalmente em mulheres (GREENDALE et al., 2003).

Conexões: revista da Faculdade de Educação Física da UNICAMP, Campinas, v. 8, n. 2, p. 68-82, maio/ago. 2010.

ISSN: 1983-9030. 
O treinamento com pesos têm sido amplamente recomendado para a população que está envelhecendo devido aos benefícios ocasionados. Mas de acordo com alguns trabalhos (ACSM, 2002; WOLFE; LEMURA; COLE, 2004), seus efeitos dependem da combinação entre o número de repetições e as séries, sobrecarga, sequiência de exercícios e intervalos de recuperação. Porém, não existe ainda um consenso sobre qual a melhor combinação entre esses componentes do treino para uma ótima relação dose-resposta para indivíduos na terceira idade.

Com isso verifica-se a necessidade de estudar a relação do treinamento com pesos com o nível de agilidade em pessoas de terceira idade, afim de, propor um protocolo de treinamento que seja mais eficaz na aquisição de melhor agilidade.

O objetivo desse estudo foi avaliar os efeitos de diferentes tipos de treinamento com pesos sobre indicadores de agilidade de mulheres idosas praticantes de atividade física regular, após dois tipos de intervenções: 12 semanas de treinamento de força (TF) e 12 semanas de treino de resistência muscular localizada (RML) e compará-los a um grupo controle (mulheres sedentárias).

\section{MATERIAIS E MÉTODOS}

\section{Sujeitos}

Foram selecionadas inicialmente 45 voluntárias, entretanto somente 42 completaram o estudo. As voluntárias dos grupos que participaram do treinamento foram consideradas fisicamente ativas por estarem inseridas em um programa de condicionamento físico com supervisão e frequiência semanal de três sessões, durante os seis meses anteriores ao estudo.

As participantes foram divididas em três grupos: Grupo Treinamento de força (GTF, $n=14$ ); Grupo Resistência Muscular Localizada (GRML, n= 14); e Grupo Controle (GC, n= 14); que não realizou nenhum tipo de atividade durante o período de treino, somente participando dos testes.

Todas as voluntárias foram informadas sobre a proposta do estudo, quais os procedimentos metodológicos e avaliações que seriam realizados e assinaram o termo de consentimento livre e esclarecido, conforme a Resolução n¹96/96 do Conselho Nacional de Saúde, de 10/10/96, aprovado pelo Comitê de Ética em Pesquisa em Seres Humanos da Universidade da Região da Campanha, Bagé/RS, (Processo de nº PQ26406).

Conexões: revista da Faculdade de Educação Física da UNICAMP, Campinas, v. 8, n. 2, p. 68-82, maio/ago. 2010.

ISSN: 1983-9030. 
Foram adotados como critérios de inclusão: idade igual ou superior a 60 anos, prática de atividade física regular a pelo menos seis meses e consideradas aparentemente saudáveis por meio do questionário Physical Activity Readiness Questionnaire PAR-Q (ACSM, 2000).

Foram excluídas da pesquisa, as voluntárias acometidas por patologias que impedissem a realização dos testes e exercícios propostos. A freqüência mínima de participação nos treinos para computação dos dados ficou estabelecida em $90 \%$.

\section{AVALIAÇÃO DO INDICADOR DE AGILIDADE}

Para a avaliação da agilidade geral foi utilizado teste de agilidade e equilíbrio dinâmico, específico para idosos, adaptado da American Alliance for Health, Physical Education, Recreation and Dance (AAHPERD) (OSNESS, 1990). Esse teste avalia somente a agilidade de membros inferiores.

Descrição do teste: como pode ser visualizado na Figura 1, à frente na cadeira foi demarcado um " $\mathrm{X}$ " sobre o solo no qual o sujeito colocava os pés; a partir do qual foram colocados dois cones bilateralmente eqüidistantes a 1,80m as laterais e 1,50m para trás, em relação à cadeira.

O avaliado partia da posição sentada na cadeira, com os calcanhares apoiados no solo, sobre o "X". Ao sinal de "pronto" e "vai", levantava-se, movia-se para a direita e circundava o cone, retornava à cadeira e sentavase. Imediatamente repetia o mesmo movimento e percurso para a esquerda completando um circuito (OSNESS, 1990). Para se certificar de que o avaliado sentava-se, o mesmo deveria retirar ligeiramente os dois pés do solo a cada vez que sentava. O avaliado foi orientado a movimentar-se o rapidamente possível sem perder o equilíbrio ou errar.

Foi realizada uma familiarização ao teste, antes da avaliação definitiva, na qual cada voluntária poderia realizar o trajeto do teste quantas vezes necessárias para um melhor entendimento.

A adaptação do teste consistiu em realizar apenas uma volta no circuito por série, ao invés de duas voltas como no protocolo original, sendo realizadas três séries separadas por um intervalo de três minutos. O tempo em cada tentativa foi registrado por meio de um cronômetro digital e expresso em segundos. Para a análise dos resultados, foi considerada a média do tempo das três tentativas. 


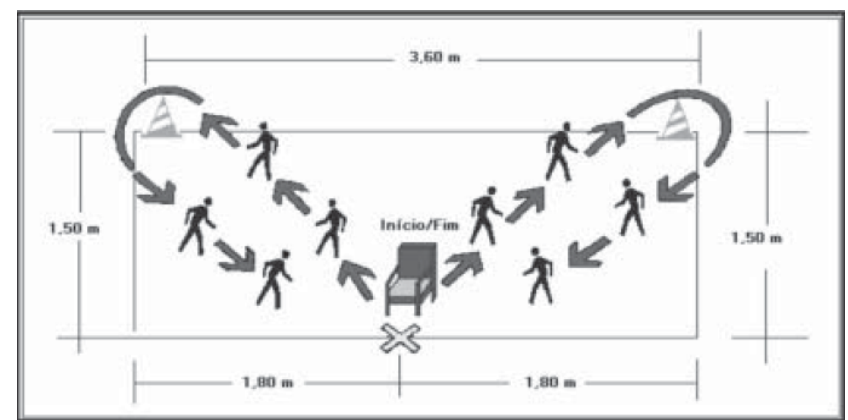

Figura 1 - Ilustração do teste de agilidade e equilíbrio dinâmico da AAHPERD (Zago ${ }^{16}$; adaptado de Osness $^{15}$ ).

\section{PROTOCOLO DE TREINAMENTO FÍSICO}

A intensidade inicial do treinamento foi igual para ambos os grupos de treinamento. Foi utilizado teste de 1RM (SILVA; FARINATTI, 2007) para a prescrição da intensidade inicial do treino, sendo realizadas duas sessões de familiarização ao teste.

Foram testados dois grupamentos musculares por dia, um exercício para membro inferior e outro para membro superior, com intervalos de 48 horas para nova tentativa.

Os testes de 1-RM foram realizados nos seguintes exercícios: supino reto barra, extensão e flexão de joelho, agachamento livre, puxador alto anterior, tríceps puley, elevação lateral e rosca direta.

Antes de cada sessão de treino foi estabelecido um aquecimento geral, que consistia em caminhada de dez minutos de duração. Logo após a sessão de treinamento foram realizados exercícios de alongamento/relaxamento.

Inicialmente a intensidade do treinamento foi de $40 \%$ da carga máxima obtida no teste de 1-RM, com três séries de quinze e vinte repetições, para GTF e GRML respectivamente, ambos com intervalos de um 1 min e 30s, segundo recomendações do ACSM (2002) ${ }^{12}$.

Após a fase de adaptação, que teve duração de 30 dias, as cargas de trabalho foram reajustadas para que a intensidade correspondesse a 80 e 60\% para GTF e GRML respectivamente, a partir dos valores obtidos em um novo teste de 1-RM.

Para os treinamentos (GTF e GRML) foram utilizados os mesmos exercícios do teste de 1-RM, acrescentando série de exercícios para panturrilha e abdominal. Para o TF adotou-se 3 séries de 5-8 repetições, com

Conexões: revista da Faculdade de Educação Física da UNICAMP, Campinas, v. 8, n. 2, p. 68-82, maio/ago. 2010.

ISSN: 1983-9030. 
intensidade da carga de $80 \%$ de 1-RM e um minuto de intervalo entre as séries. Para a RML foram fixadas 3 séries de 15 a 20 repetições e intervalo de 1 min entre as séries.

A execução dos exercícios obedecia à ordem dos maiores para os menores grupamentos musculares, sempre alternados por segmentos. A progressão subseqüente de cargas foi ajustada de acordo com a subjetividade do avaliador quando a voluntária sentia muita facilidade em executar o número de repetições em cada série.

O GC não realizou exercícios físicos de forma regular e sistematizada durante o período do estudo, realizando somente as avaliações em conjunto com os outros grupos. Todas voluntárias deste grupo foram consideradas fisicamente inativas por não participarem de atividade física regular por um período de seis meses anteriores à pesquisa.

\section{ANÁLISE DOS DADOS}

Foi utilizado o pacote estatístico STATISTICA ${ }^{\mathrm{TM}}$. A normalidade dos dados foi verificada através do teste de Shapiro-Wilk. A análise de variância (ANOVA) para medidas repetidas foi empregada para as comparações entre os grupos nos diferentes momentos do estudo (inicial e final). O teste post hoc de SCHEFFÉ para comparações múltiplas foi empregado para a identificação das diferenças nos tempos em que os valores de $\mathrm{F}$ encontrados foram superiores ao critério de significância estatística estabelecida $(p<0,05)$.

\section{RESULTADOS}

Não foram encontradas diferenças estatisticamente significantes para a idade $(\mathrm{GTF}=68,0 \pm 2,7 ; \mathrm{GRML}=$ $67,2 \pm 2,6 ; \mathrm{GC}=68,2 \pm 3,9$ ) quando comparados os grupos estudados apontando para uma homogeneidade da amostra quanto a faixa etária.

Na Tabela 1, são apresentados os resultados de cada grupo no teste de agilidade. O GTF, apresentou diminuição significante dos valores entre os momentos. Não foram encontradas modificações significantes no tempo de realização do teste para o GRML e GC. Na comparação entre os grupos o GTF apresentou diferença significativa quando comparado ao GRML e GC. Não foram observadas diferenças significantes entre GRML e GC. 
Tabela 1 - Valores Médios e Desvios Padrões do Tempo Médio de Teste de Agilidade Expresso em Segundos, dos Grupos Estudados.

\begin{tabular}{lcccc}
\hline Grupos & n & Pré & Pós & p \\
\hline GTF & 14 & $11^{\prime \prime}, 97 \pm 2,40$ & $9 ", 64 \pm 1,70^{* \dagger \ddagger}$ & 0,02 \\
GRML & 14 & $12 ", 16 \pm 1,25$ & $11 ”, 43 \pm 1,11$ & 0,939 \\
GC & 14 & $12 ”, 47 \pm 1,32$ & $12 ”, 41 \pm 1,21$ & 0,975
\end{tabular}

* Diferença significativa do pré para o pós, ${ }^{\dagger}$ diferença significativa do momento pós entre GTF e

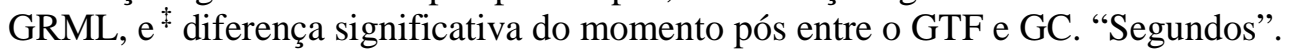

A Figura 2, apresenta a modificação percentual de cada grupo do momento pré para o momento pós (percentual de modificação). Somente foram observadas diferenças significantes na comparação entre os grupos GTF e GC (p<0,005).

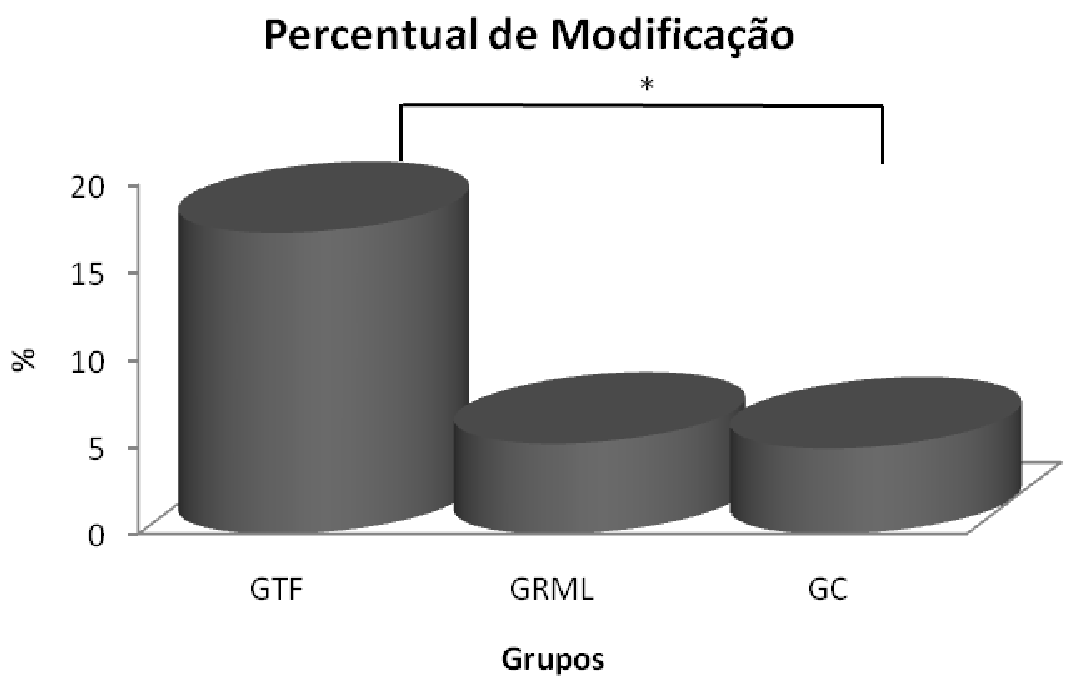

Figura 2 - Percentual de modificação entre os grupos.

* Diferença significante entre GTF e GC.

\section{DISCUSSÃO}

Esta pesquisa buscou avaliar os efeitos de diferentes tipos de treinamento (TF e RML) sobre indicadores de agilidade de mulheres idosas praticantes de atividade física regular, comparado ao GC (mulheres sedentárias).

O TF diferenciou-se da RML pela intensidade do treinamento, visto que a carga pode ser modificada pelo volume do treinamento e pela intensidade dos exercícios. Neste estudo consideramos o TF como de alta intensidade ( $80 \%$ de 1-RM) e o RML de baixa intensidade (60\% de 1-RM). Entretanto, assumindo-se a 
especificidade de cada protocolo de treino, ambos podem trazer benefícios para a saúde de idosos (SILVA, FARINATTI, 2007).

Como preconizado pelo ACSM (2002), os conceitos de força e de RML baseiam-se na quantidade de repetições realizadas em uma série, no intervalo entre as séries e na carga deslocada. No presente estudo, foram propostos dois protocolos de treinos evidenciando as diferenças entre força e RML, para compará-los quanto à aquisição de agilidade. Para o GTF foi prescrito número baixo de repetições com uma carga elevada, caracterizando treino de força e para o GRML foi prescrito número elevado de repetições e baixa carga, caracterizando treino de RML, a fim de diferenciar os protocolos.

A repetição do teste de 1-RM para progressão de carga torna-se inviável, pois pode comprometer a intensidade do treino, cuja que a intensidade é superior a da prescrição dos treinamentos e além de subestimar ou superestimar alguns exercícios, podendo ser este um fator limitante do estudo.

Ao final do período de intervenção somente o GTF mostrou melhora significante da agilidade. O GRML mostrou modificações, com diminuição do tempo médio de teste de agilidade embora sem diferenças estatisticamente significativas, e ainda o GC não apresentou nenhuma alteração importante.

Por se tratar de grupos que já estavam inseridos em um programa regular de atividade física, a modificação do tipo de treinamento (RML para força), pode ter sido um fator determinante para os resultados encontrados, visto que a intensidade do treino para o GTF foi maior em comparação com o GRML.

A resposta encontrada no GTF mostra que mesmo num período curto de intervenção ocorrem importantes mudanças no indicador de agilidade aplicado que podem contribuir para a melhoria da capacidade funcional de mulheres idosas.

Estudo realizado com mulheres de idade inferior a 75 anos sugere que a massa de gordura pode ser o principal determinante para a diminuição da capacidade funcional em mulheres até esta idade, enquanto que a sarcopenia torna-se determinante após este período (LEBRUN et al., 2006).

A associação entre aspectos de desempenho neuromusculares, força e massa muscular de membros inferiores foram analisadas em outro estudo (VISSER et al., 2000), por meio de testes muito similares ao aplicado no presente estudo. Através da avaliação da massa muscular e o desempenho neuromotor dos membros inferiores foi verificada associação entre a reduzida força muscular, (mas não entre massa muscular), e o pior

Conexões: revista da Faculdade de Educação Física da UNICAMP, Campinas, v. 8, n. 2, p. 68-82, maio/ago. 2010. 
desempenho neuromotor dos membros inferiores em homens e mulheres idosas, sugerindo, que a força parece ser mais importante do que a massa muscular para o desempenho neuromotor nas atividades cotidianas, como o andar e o levantar-se da cadeira (VISSER et al., 2000).

Segundo o ACSM (2002), a força muscular é um importante componente na habilidade do indivíduo em parar e mudar de direção rapidamente e recomenda um protocolo mais específico para a aquisição de agilidade, assim como força e potência.

Nesse sentido, os resultados do estudo de Marcovic (2007), mostram correlação positiva entre o pico de força, potência e testes de agilidade.

Corroborando aos resultados deste estudo, encontramos uma pesquisa (WOOD et al., 2001), que comparou os efeitos sobre a agilidade de três diferentes tipos de treinamento: aeróbio, com pesos e concorrente durante 12 semanas de intervenção, em homens e mulheres idosas. Os resultados encontrados mostraram melhora significativa dos indicadores de agilidade nos três grupos avaliados, comparados a um grupo controle. Essa divergência com os dados do nosso estudo pode ser atribuída à condição inicial dos voluntários, que no referido estudo (WOOD et al., 2001) eram inicialmente não ativas.

O comportamento dos níveis de agilidade nos idosos pode ser influenciado no processo de envelhecimento pela diminuição da força e da flexibilidade, entre outras, mudanças que podem ser prevenidas pelo treinamento com pesos (GOBBI et al., 2005).

Respostas positivas foram encontradas em relação ao incremento na velocidade de levantar-se da cadeira e caminhar três metros como também pela realização de algumas atividades da vida diária, instrumentais e não instrumentais, aplicando um programa de treinamento de força de baixa intensidade, com duração de 10 semanas, em sujeitos maiores de 65 anos, sendo esses efeitos mantidos por pelo menos seis meses (WESTHOFF; STEMMERIK; BOSHUIZEN, 2000).

Comparando o treinamento do estudo anterior (WESTHOFF; STEMMERIK; BOSHUIZEN, 2000) ao treinamento do GRML, podemos considerar ambos de baixa intensidade. Visto que não encontramos melhoras significativas dos indicadores de do GRML, podemos assumir que o treinamento de baixa intensidade não contribuiu para a melhora deste indicador, contribuindo apenas para a manutenção da mesma no grupo ativo por nós estudado.

Conexões: revista da Faculdade de Educação Física da UNICAMP, Campinas, v. 8, n. 2, p. 68-82, maio/ago. 2010.

ISSN: 1983-9030. 
O efeito de um programa de oito meses de exercícios físicos, sobre aptidão física e capacidade funcional de mulheres saudáveis, subdivididas em grupos etários de 65, 75 e 85 anos de idade, apresentou melhoras na potência aeróbia e na velocidade de andar, contribuindo para a melhoria da aptidão física total, evidenciando claramente os benefícios da atividade física independentemente das idades estudadas (PUGGAARD, 2003).

Estudos de revisão demonstram que a prática regular de atividade física pode reduzir a incidência de quedas de $20 \%$ a $40 \%$, sugerindo ser essa mais uma razão para que as pessoas idosas mantenham-se ativas fisicamente (NESLON et al., 2007).

Por outro lado, a diminuição da força muscular pode influenciar diretamente a capacidade funcional dos indivíduos, reduzindo a independência para realizar as tarefas da vida diária, principalmente em indivíduos idosos. Assim, os benefícios do treinamento com pesos para manutenção e aumento dos níveis de força estão bem fundamentados na literatura e podem ser aplicados a qualquer população, podendo influenciar diretamente na capacidade funcional e qualidade de vida dos praticantes (BENEDETTI, 2007).

Para os idosos o treinamento com pesos pode trazer muitos benefícios como o aumento da força e potência muscular, a diminuição das dificuldades em realizar as tarefas diárias, o aumento do gasto energético, mudanças positivas na composição corporal e pode ser um incentivo à participação em programas de atividade física (SILVA et al., 2006).

No entanto vale ressaltar uma dificuldade encontrada nesse estudo foi comparar os valores encontrados no teste de agilidade com os já publicados na literatura, devido à adaptação utilizada (ZAGO, 2002;GOBBI et al., 2005; BENEDETTI, 2007).

Essa adaptação foi necessária, pois ao final de cada volta a maioria das voluntárias demoravam a iniciar a segunda volta, ou esqueciam-se de realizar a mesma, comprometendo o tempo de realização do teste.

\section{CONCLUSÃO}

A partir dos resultados encontrados conclui-se que o TF mostrou melhor influência, em relação ao treino de RML, para melhora do indicador de agilidade em mulheres idosas fisicamente ativas.

Desta forma o TF pode contribuir, para a melhora da agilidade, entretanto, a intensidade do treino pode ter sido fator determinante para provocar alterações benéficas somente no GTF, sugerindo que a prescrição de

Conexões: revista da Faculdade de Educação Física da UNICAMP, Campinas, v. 8, n. 2, p. 68-82, maio/ago. 2010.

ISSN: 1983-9030. 
treino com intensidades mais elevadas causa melhores adaptações para incremento da agilidade em idosa.

\section{REFERÊNCIAS}

ACSM. American College of Sports Medicine. ACSM's guidelines for exercise testing and prescription. In: POWERS, S. K., HOWLEY, E. T. Fisiologia do exercício: teoria e aplicação ao condicionamento e ao desempenho. Barueri: Manole; 2000.

. Position stand: progression models in resistance training for healthy adults. Med Sci Sports Exerc, v. 34, p. 364-380, 2002.

. Diretrizes do ACMS para os testes de esforço e sua prescrição. Rio de Janeiro: Guanabara Koogan; 2003.

BAUMGARTNER, R. N. et al. Epidemiology of sarcopenia among the elderly in New Mexico. Am $J$ Epidemiol, v. 147, n. 8, p. 755-763, 1998.

BENEDETTI, T. R. B. et al. Valores normativos de aptidão funcional em mulheres de 70 a 79 anos. Rev Bras Cineantropom Desem Hum, v. 9, n. 1, p. 28-36, 2007.

FERREIRA, L.; GOBBI, S. E. Agilidade geral e agilidade de membros superiores em mulheres de terceira idade treinadas e não treinadas. Rev Bras de Cineantropom Desem Hum, v. 5, n. 1, p. 46-53, 2003.

GOBBI, S. et al. Bases teóricos-práticas do condicionamento físico. Rio de Janeiro: Guanabara Koogan; 2005.

GREENDALE, G. A. et al. Sport and home physical activity are independently associated with bone density. Med Sci Sports Exerc, v. 35, n. 3, p. 506-512, 2003.

GURALNIK, J. M.; LACROIX, A. Z. Assessing physical function in older populations. In: WALLACE, R. B.; WOOLSON, R. F. (Ed.). The epidemiologic study of the elderly. New York: Oxford University Press, 1992. p. 159-81.

HUNTER, G. R.; MCCARTHY, J. P.; BAMMAN, M. M. Effects of resistance training on older adults. Sports Med, v. 34, n. 5, p. 329-348, 2004.

IBGE. Instituto Brasileiro de Geografia e Estatística. Brasil, 2004. Disponível em: www.ibge.org.br. Acesso em: 16 mar. 2007.

KISS, M. A. P. D. Esporte e exercício avaliação e prescrição. São Paulo: Roca, 2003.

LATHAM, N. K. et al. Systematic review of progressive resistance strength training in older adults. $J$ Geront Med Sci, v. 54, p. 48-61, 2004.

Conexões: revista da Faculdade de Educação Física da UNICAMP, Campinas, v. 8, n. 2, p. 68-82, maio/ago. 2010.

ISSN: 1983-9030. 
LEBRUN, C. E. I. et al. Fat mass rather than muscle strength is the major determinant of physical function and disability in postmenopausal women younger than 75 years of age. Menopause, v. 3, n. 13, p. 474-481, 2006.

MARCOVIC, G. Poor relationship between strength and power qualities and agility performance. $J$ Sports Med Phys Fitness, v. 47, n. 3, p. 276-283, 2007.

MATSUDO, S. M.; MATSUDO V. K. R.; BARROS NETO, T. L. Impacto do envelhecimento nas variáveis antropométricas, neuromotoras e metabólicas da aptidão física. Rev Bras Cienc Mov, v. 8, n. 4, p. 21-32, 2000. MATSUDO, S. M. et al. Evolução do perfil neuromotor e capacidade funcional de mulheres fisicamente ativas de acordo com a idade cronológica. Rev Bras Med Esporte, v. 9, n. 6, p. 365-376, 2003.

NELSON, M. E. et al. Physical activity and public health in older adults: recommendation from the American College of Sports Medicine and the American Heart Association. Med Sci Sports Exerc, v. 39, n. 8, p. 1435$1445,2007$.

OSNESS, W. H. Functional fitness assessment for adults over 60 years. Reston: AAHPERD, 1990.

PUGGAARD, L. Effects of training on functional performance in 65,75 and 85 year-old women: experiences deriving from community based studies in Odense, Denmark. Scand J Med Sci Sports, v. 13, n. 1, p. 70-76, 2003.

ROSA, T. E. C. et al. Fatores determinantes da capacidade funcional entre idosos. Rev Saúde Pub; v. 37, n. 1, p. 40-48, 2003.

SILVA, C. M. et al. Efeito do treinamento com pesos, prescrito por zona de repetições máximas, na força muscular e composição corporal em idosas. Rev Bras Cineantropom Desem Hum, v. 8, n. 4, p. 39-45, 2006.

SILVA, N. L.; FARINATTI, P. T. V. Influência de variáveis do treinamento contra-resistência sobre a força muscular de idosos: uma revisão sistemática com ênfase nas relações dose-resposta. Rev Bras Med Esporte, v. 13, n. 1, p. 60-66, 2007.

VISSER, M. et al. Skeletal muscle mass and muscle strength in relation to lower-extremity performance in older men and women. J Am Geriatr Soc, v. 48, n. 4, p. 381-386, 2000.

WESTHOFF, M.; STEMMERIK, L.; BOSHUIZEN, H. Effects of a low-intensity strength-training program on knee-extensor strength and functional ability of frail older people. JAPA, n. 8, p. 325-342, 2000.

WHO. World population prospects the 2004 revision. Disponível em: www.Un.Org/Esa/Population/Publications/Wpp2004/2004highlights_Finalrevised.Pdf> Acesso em: 20 mar. 2007.

Conexões: revista da Faculdade de Educação Física da UNICAMP, Campinas, v. 8, n. 2, p. 68-82, maio/ago. 2010.

ISSN: 1983-9030. 
WOLFE, B. L.; LEMURA, L. M.; COLE, P. I. Quantitative analysis of single- vs. multiple set programs in resistance training. J Strength Cond Res, v. 18, p. 35-47, 2004.

WOOD, R. H. et al. Concurrent cardiovascular and resistance training in healthy older adults. Med Sci Sports Exerc, v. 33, n. 10, p. 1751-1758, 2001.

ZAGO, A. S. Relação do nível de aptidão funcional com os fatores de risco de doenças coronarianas associadas à bioquímica sangüínea e a composição corporal em mulheres ativas de 50 a 70 anos. 2002. Dissertação (Mestrado em Ciências da Motricidade Humana) - Instituto de Biociências, Universidade Estadual Paulista, Rio Claro, 2002.

\section{MIGUEL SOARES CONCEIÇÃO}

Núcleo de pesquisa em Atividade Física, Exercício Físico e Saúde, Curso de Educação Física, URCAMP-Bagé/RS

\section{VALÉRIA BONGANHA}

Pós-Graduação FEF/UNICAMP

\section{FÁBIO BITENCOURT LEIVAS}

Núcleo de pesquisa em Atividade Física, Exercício Físico e Saúde, Curso de Educação Física, URCAMP-Bagé/RS

\section{GIANCARLO B. M. BRUNO}

Núcleo de pesquisa em Atividade Física, Exercício Físico e Saúde, Curso de Educação Física, URCAMP-Bagé/RS

\section{FELIPE CASSARO VECHIN}

Estagiário do Laboratório de Fisiologia do Exercício - FISEX/UNICAMP

\section{MARA PATRÍCIA TRAINA CHACON-MIKAHIL}

Docente Faculdade de Educação Física/UNICAMP

\section{VERA APARECIDA MADRUGA}

Docente Faculdade de Educação Física/UNICAMP

\section{Referência do artigo}

\section{ABNT}

CONCEIÇÃO, M. S. et al. Treinamento com pesos e indicadores de agilidade de mulheres idosas. Conexões, v. 8, n. 2, p. 68-82, 2010. 
APA

CONCEIÇÃO, M. S., BONGANHA, V., LEIVAS, F. B., BRUNO, G. B. M., VECHIN, F. C., CHACON-MIKAHIL, M. P. T. et al. (2010). Treinamento com pesos e indicadores de agilidade de mulheres idosas. Conexões, 8(2), 6882.

\section{VANCOUVER}

CONCEIÇÃO MS, BONGANHA V, LEIVAS FB, BRUNO GBM, VECHIN FC, CHACON-MIKAHIL MPT, MADRUGA VA. Treinamento com pesos e indicadores de agilidade de mulheres idosas. Conexões, 2010; 8 (2): 68-82.

Recebido em: abr./2010

Aceito para publicação em: jun./2010 